\title{
Review of: "Comparison between intervertebral oblique lumbar interbody fusion and transforaminal lumbar interbody fusion: a multicenter study"
}

\author{
Yoshihisa Kotani
}

Potential competing interests: The author(s) declared that no potential competing interests exist.

Utilizing the local multicenter database, this study compared the clinical and radiologic results between Oblique Lateral Interbody Fusion (OLIF) with posterior percutaneous screws (PPS) and open Transforaminal Interbody Fusion (TLIF). Although many reports highlighted less postoperative invasiveness of Lateral Interbody Fusion (LIF) surgery, the authors evaluated the symptomatic and asymptomatic adjacent segment diseases (ASD) over three-year follow-up, providing a valuable data in spine surgery field. Their results demonstrated increased frequency of asymptomatic ASD of $21 \%$ vs $7 \%$ in OLIF group and TLIF group, respectively, however, symptomatic ASD and reoperation rate were comparable. It was questionable that Figure 4 depicted the asymtomatic ASD value of $13 \%$ in OLIF group, which was far different from descriptive result of $21 \%$ in the paper.

Even in many comparative study reported (1-5), there were many variations in surgical modality in terms of whether position change was required, whether TLIF procedure was open or minimally invasive surgery (MIS), and graft bone materials. In this study, PPS procedure was performed in prone after reposition, and TLIF was a full open procedure.

The advantage of LIF fully demonstrates the use of lateral position surgery without position change, in terms of total operation time, estimated blood loss and consumable costs $(4,5)$.

Although the authors failed to demonstrate the advantage of OLIF vs open TLIF, some recent reports successfully demonstrated the advantage of OLIF with lateral position surgery being comparable even to MIS-TLIF (not open TLIF), as well as less correction loss of disc height and restoration of segmental lordosis $(4,5)$.

Although many discussion are still underway in this filed, further discussion is necessary whether indirect decompression with LIF increases ASD or not in long-term, as well as total invasiveness, infection rate and etc. I finally appreciated the authors' great publication for readers.

1. Kim, J. S. et al. Mini-transforaminal lumbar interbody fusion versus anterior lumbar interbody fusion augmented by percutaneous pedicle screw fxation: A comparison of surgical outcomes in adult lowgrade isthmic spondylolisthesis. J. Spinal Disord. Tech. 22, 114-121 (2009).

1. Saadeh, Y. S. et al. Comparison of segmental lordosis and global spinopelvic alignment afer single-level 
lateral lumbar interbody fusion or transforaminal lumbar interbody fusion. World Neurosurg. 126, e1374-e1378 (2019).

2. Jin, J. et al. Comparative study of the diference of perioperative complication and radiologic results: MISDLIF (Minimally invasive direct lateral lumbar interbody fusion) versus MIS-OLIF (Minimally invasive oblique lateral lumbar interbody fusion). Clin. Spine Surg. 31, 31-36 (2018).

3. Koike, Y. et al. Comparison of outcomes of oblique lateral interbody fusion with percutaneous posterior fxation in lateral position and minimally invasive transforaminal lumbar interbody fusion for degenerative spondylolisthesis. Asian Spine J. 15, 97-106 (2021).

4. Kotani $Y$, et al. Clinical and radiologic comparison of anterior-posterior single-position lateral surgery versus MIS-TLIF for degenerative lumbar spondylolisthesis.

J Orthop Sci. 2020 Dec 16 (E-pub ahead of print) 